\title{
The effect of radar-based QPE on the Fractions Skill Score used at the $Q P F$ verification
}

\author{
P. Zacharov and D. Rezacova \\ Institute for Atmospheric Physics, Prague, Czech Republic \\ Received: 30 October 2009 - Revised: 16 March 2010 - Accepted: 17 March 2010 - Published: 30 March 2010
}

\begin{abstract}
In this paper we show the influence of gauge adjustment technique, applied to radar-based rainfalls, on the results of QPF verification. The results were obtained for four convective events which produced heavy local rainfalls and caused local flash floods at the Czech territory. $\mathrm{Nu}-$ merical weather prediction model COSMO was run to obtain rainfall forecast and Fractions Skill Score was employed in the QPF verification. Three different radar-based quantitative precipitation estimates (QPE) were used for the verification and the verification results were compared. The QPE data sets consisted of: (a) raw radar-based rainfall values, (b) gauge corrected radar-based rainfalls with a simple domain-wide correction, and (c) radar-based rainfalls with a pixel related gauge adjustment. The results indicate small difference in area-related verification results and prove that the simple domain wide correction technique is sufficient for applying radar-based rainfalls as the verification data.
\end{abstract}

\section{Introduction}

The use of radar data appears to be important especially for the estimation of local heavy convective rainfalls which can cause local flash floods. Because of their small area extents, such rainfalls are difficult to be registered by standard gauge networks. However, a gauge adjustment of radar rainfall values is needed because of the nature of radar measurements. The density and positions of gauges and an adjustment technique can influence the resulted Quantitative Precipitation Estimation (QPE). It is especially valid if we determine the convective rainfall values of shorter accumulation periods. Apart from a direct QPE application in hydrological predictions and nowcasting, we need the QPE to verify the Quantitative Precipitation Forecast (QPF). QPF verification by radar-based data were used as verification data e.g. by Ebert (2008), who compared various so called fuzzy verification techniques. Case studies dealing with precipitation forecast verification and using ground radar precipitation adjusted by other sensors mention differences in verification results after using different verification data (Tartaglione et al., 2006; Casaioli et al., 2006).

In this paper, we summarize the results obtained at studying the influence of adjustment technique on the QPF verification by radar-based precipitation. The results were obtained for four convective events which occurred at the territory of the Czech Republic (CR). The events, which produced heavy local rainfalls and caused local flash floods, are described in Rezacova et al. (2009). The radar data fields of individual cases prove different area extents and structures. In order to obtain the QPF we used nonhydrostatic numerical weather prediction model COSMO with the resolution of $2.8 \mathrm{~km}$ (Rezacova et al., 2009, Zacharov and Rezacova, 2009). The forecast skill for individual events is commented in Rezacova et al. (2009). The verification results refer to the QPE and QPF which correspond to the $1 \mathrm{~h}, 3 \mathrm{~h}$ and $6 \mathrm{~h}$ rainfall values.

The paper is divided in four parts. Part 2 presents the adjustment techniques starting with daily radar-based QPE values. In order to obtain the QPE at shorter rainfall accumulation we applied a simple area-related correction and a pixel-oriented adjustment. Part 3 shows how the verification of QPF reflects the adjustment technique used. The verification applies Fractions Skill Score as a representative of so called "fuzzy" verification. Part 4 summarizes the results and concludes the paper.

\section{Correspondence to: D. Rezacova}

(rez@ufa.cas.cz)

Published by Copernicus Publications on behalf of the European Geosciences Union. 


\section{Radar-based precipitation}

Radar data from the two radars of the CZRAD network were used to determine the rough estimates of rainfalls. The two radars are Doppler and C-band radars operated by the Czech Hydro-meteorological Institute (CHMI). Radar reflectivity datasets are provided in $10 \mathrm{~min}$ intervals and operational correction procedures are applied in the CHMI (Novák, 2007).

In order to determine the rainfall values, the resulting PPI reflectivity values are transformed to radar pixels with a horizontal resolution of $1 \mathrm{~km}$ at the level of $2000 \mathrm{~m}$ a.m.s.l. Radar reflectivity $z\left(\mathrm{~mm}^{6} \mathrm{~m}^{-3}\right)$ is transformed into rain rate $R(\mathrm{~mm} / \mathrm{h})$ according to the common MP relationship $z=$ $200 R^{1.6}$. Accumulated hourly radar-based rainfalls were determined by integration of corresponding rain rates.

Several gauge adjustment techniques were tested with the radar data from the Czech radar network CZRAD (e.g. Zacharov et al., 2004). Present operational adjustment technique, applied in the CHMI, uses data from automatic gauges, which provide hourly rainfall measurements. However, the number of automatic gauges is limited and the gauge spatial density over the Czech territory varies. That is the reason why we apply the daily rainfall values from more than 800 gauges to the verification of the QPF for heavy local convective rainfalls.

In this paper we study the influence of gauge adjustment techniques on the results of QPF verification (see part 3). Firstly we applied the raw radar-based values without gauge adjustment. Secondly we applied gauge adjustment technique described in Sokol (2003) and Rezacova et al. (2007), which used daily precipitation data from gauges, in order to determine adjusted daily values. The adjusted daily radar precipitation was determined separately for both radars. Within the overlapping areas the maximum of two daily values was taken into account.

After obtaining the adjusted daily precipitation amounts in the radar pixels, we derived corrected hourly precipitation totals by multiplying the raw radar hourly values by the relative daily precipitation amounts. The simple correction over the verification domain followed the formula

$$
R(i)_{\text {cor_lh }}=R(i)_{\text {raw_l }} \frac{\sum_{j=1}^{\mathrm{N}} R(j)_{\text {adj_day }}}{\sum_{j=1}^{\mathrm{N}} R(j)_{\text {raw_day }}},
$$

where $R(i)_{\text {cor_- } 1 \mathrm{~h}}$ is the gauge corrected $1 \mathrm{~h}$ radar rainfall over the pixel $i$ and $R_{\text {raw }} 1 \mathrm{~h}$ is the corresponding rough $1 \mathrm{~h}$ radar rainfall. The $R_{\text {adj_day }}$ and $R_{\text {raw_day }}$ are the gauge adjusted and raw daily pixel values, respectively, and the sums cover the radar pixels inside the verification domain. The above correction technique uses a strong simplification as it applies a constant correction factor over the entire verification
Table 1. Characteristics of radar-based and forecast rainfall [mm] with accumulation of $6 \mathrm{~h}$ (16:00 UTC-22:00 UTC). The mean (oX_m) and maximum $\left(\mathrm{oX} \_\mathrm{x}\right)$ observed values show grid rainfall values over the entire verification domain. $X=1,2,3$ refers to the verification by using raw radar data, area corrected radar data and pixel-adjusted radar data, respectively. Similarly, f_m and f_x give the mean and maximum forecast rainfall.

\begin{tabular}{lcccc}
\hline rainfall type & \multicolumn{4}{c}{ event datum (yymmdd) } \\
& 020713 & 020715 & 040610 & 050523 \\
\hline o1_m & 6.2 & 0.6 & 2.5 & 5.7 \\
o1_x & 101.5 & 69.0 & 32.7 & 95.6 \\
o2_m & 4.4 & 0.5 & 2.2 & 8.0 \\
o2_x & 73.6 & 76.8 & 30.6 & 135.5 \\
o3_m & 6.1 & 0.6 & 3.5 & 9.6 \\
o3_X & 121.8 & 169.0 & 66.0 & 140.2 \\
f_m & 6.0 & 1.0 & 1.1 & 3.7 \\
f_x & 106.8 & 36.3 & 42.8 & 50.9 \\
\hline
\end{tabular}

domain. That is why an alternative pixel-related adjustment in the form

$R(i)_{\text {adj_- } 1 \mathrm{~h}}=R(i)_{\text {raw_1h }} \frac{R(i)_{\text {adj_day }}}{R(i)_{\text {raw_day }}}$

was applied locally in each radar pixel. The gauge adjusted hourly rainfalls were then interpolated into the model grid points and the rainfalls of a given accumulation were acquired by summing up the adjusted hourly rainfalls. Table 1 shows basic characteristics of raw, corrected and adjusted radar rainfalls together with corresponding forecast values (see part 3).

\section{QPF verification using radar-based precipitation}

The NWP model COSMO was used to forecast the precipitation (Rezacova et al., 2009; Zacharov and Rezacova, 2009). The COSMO model was run in two steps. In the first step the driving COSMO was implemented over a large part of Europe with a horizontal resolution of $11 \mathrm{~km}$, with initial and lateral boundary conditions derived from ECMWF data. The model integration started at 00:00 UTC and finished after $24 \mathrm{~h}$. In the second step the driven COSMO was run over the CR with a horizontal resolution of $2.8 \mathrm{~km}$ starting at 06:00 UTC. Initial and boundary conditions were derived from the driving COSMO runs and were available at each hour of the integration time. Verification was made over the verification domain covering the $\mathrm{CR}$. 

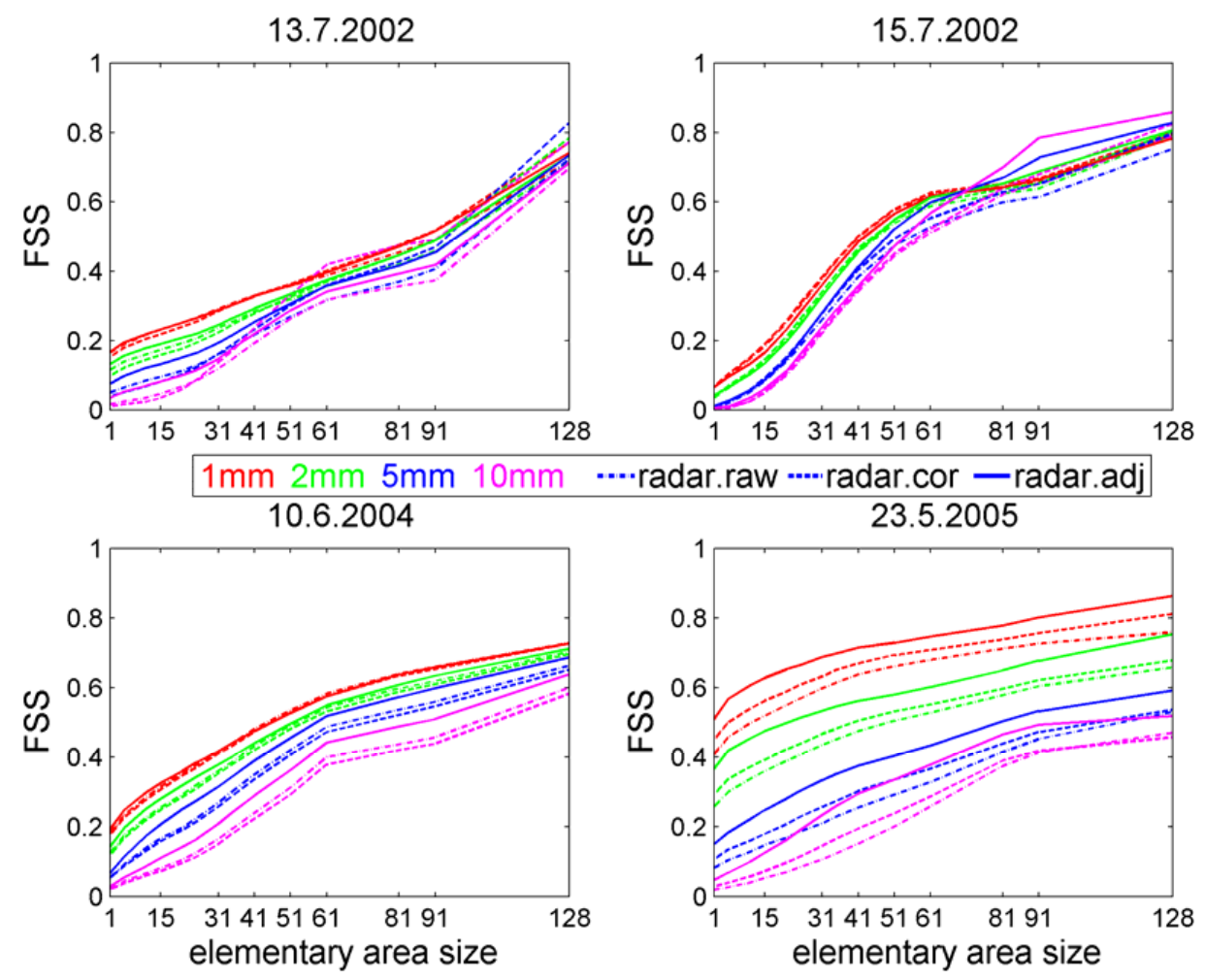

Fig. 1. Mean FSS against the spatial scale for $3 \mathrm{~h}$ rainfall. The scale (horizontal axis) is marked by the number of grid points in the side of the square elementary area (marked by $N_{g}$ in the text). The mean FSS were calculated over all hourly model outputs given threshold (indicated in the legend). They were determined from raw radar data, area corrected radar data and pixel-adjusted radar data (see the legend). The label 128 on the horizontal axis marks the elementary area corresponding to the entire domain.

In this study, we demonstrate the effect of gauge adjustment on the QPF verification by applying Fractions Skill Score (FSS). The FSS was defined by the formula (see e.g. Roberts and Lean, 2008; Ebert, 2008)

$\mathrm{FSS}=1-\frac{\frac{1}{\mathrm{~N}} \sum_{\mathrm{N}}\left(\mathrm{P}_{\mathrm{F}}-\mathrm{P}_{\mathrm{O}}\right)^{2}}{\frac{1}{\mathrm{~N}} \sum_{\mathrm{N}} \mathrm{P}_{\mathrm{F}}{ }^{2}+\frac{1}{\mathrm{~N}} \sum_{\mathrm{N}} \mathrm{P}_{\mathrm{O}}{ }^{2}}$

where $\mathrm{P}_{x}$ (for $x=\mathrm{F}, \mathrm{O} ; \mathrm{F}$ - forecast, $\mathrm{O}$ - observation) is the fractional coverage of an elementary area by rainfall that exceeds a given threshold value, and $\mathrm{N}$ gives the number of grid points in the verification domain. The denominator is a version of the Fraction Brier Score (FBS) in which fractions are compared and it gives the worst possible FBS in which there is no overlap of non-zero fractions. In order to study the effect of upscaling on the FSS values, the $\mathrm{P}_{x}$ values were determined over a square elementary areas centered in each grid point. The elementary area size was given by the number of model grid points in the square side $\left(N_{g}\right)$. The calculations were done for $N_{g}=1,5,11,15,21,25,31$, $35,41,51,61,81,91$ grid points and for the whole verification domain $(165 \times 95$ grid points). The use of FSS corresponds to the application of the so-called "fuzzy" verification techniques and causes some relaxation of the requirement of exact matches between grid point (or area) forecasts and observations (Ebert, 2008).

In the FSS calculation we used threshold values of 1, 2, 5, and $10 \mathrm{~mm}$ and we performed the evaluation separately for 1 , 3 , and $6 \mathrm{~h}$ rainfalls. The rainfalls were determined with a time step of $1 \mathrm{~h}$ starting after $7 \mathrm{~h}$ (13:00 UTC-14:00 UTC), $5 \mathrm{~h}$ (11:00 UTC-14:00 UTC), and 4h (10:00 UTC-16:00 UTC) of integration time for $1 \mathrm{~h}, 3 \mathrm{~h}$, and $6 \mathrm{~h}$ rainfalls, respectively. The last considered rainfalls corresponded to the time periods 21:00 UTC-22:00 UTC ( $1 \mathrm{~h}$ rainfalls), 19:00 UTC22:00 UTC ( $3 \mathrm{~h}$ rainfalls), and 16:00 UTC-22:00 UTC (6h rainfalls). This means that the FSS values were computed for 9 ( $1 \mathrm{~h}$ rainfalls), 9 ( $3 \mathrm{~h}$ rainfalls), and 7 (6 h rainfalls) rainfall fields at every event. The whole set of FSS values comprised 504 values ( $1 \mathrm{~h}$ and $3 \mathrm{~h}$ rainfalls) and 392 values (6h rainfalls) for each of four convective events.

In Fig. 1 we present the mean FSS values against the spatial scale for $3 \mathrm{~h}$ rainfall. There is a general increase in FSS with increasing elementary area and with decreasing rainfall threshold at all events and verification data sets. Events from 2002 show negligible effect of the type of verification data. The same is valid for 2004 event and higher thresholds. The 2005 event shows higher FSS values for gauge adjusted radar 


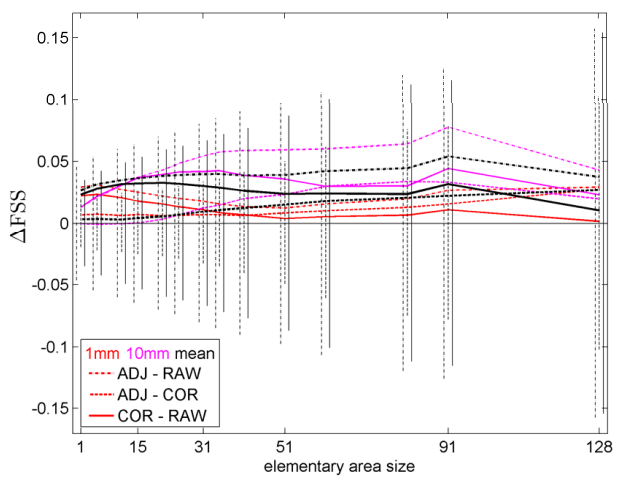

Fig. 2. The mean FSS difference ( $\triangle \mathrm{FSS})$ against the spatial scale for $3 \mathrm{~h}$ rainfall. The scale (horizontal axis) is marked by the number of grid points at the side of the square elementary area. Like in the Fig. 1, the label 128 marks the entire domain. The mean $\Delta \mathrm{FSS}$ values were calculated over all events and integration times. They refer to various radar-based data types as indicated in the legend. The color curves correspond to the thresholds $1 \mathrm{~mm}$ and $10 \mathrm{~mm}$. The black curves show means over all the threshold values considered $(1,2,5$, and $10 \mathrm{~mm})$ and the black vertical lines indicate corresponding standard deviation values.

rainfalls. Rezacova et al. (2009) showed the poor quality of the forecast for 2005 event. The predicted convective system was situated to the west part of the CR while the real precipitation system developed largely over the south part of the CR. As some gauges reported light local rainfall in the area of forecast precipitation, the adjusted data provide the best results. Generally the highest FSS values are provided by local radar adjustment followed by the domain-wide radar correction.

Figure 2 transforms the results from Fig. 1 emphasizing the difference between FSS for various radar data types. All the mean difference values are positive, which means better FSS values after correcting or adjusting the radar-based rainfalls. However, the mean FSS differences are of the order of 0.01. Apart from mean differences it indicates the standard deviation of the whole difference distribution. We can see that the standard deviation of differences increases with elementary area size because of the inclusion of all different events. However the standard deviation values stay in the limits of 0.15 in absolute value.

The high correlation between FSS values obtained from different data is indicated in Fig. 3. The figure shows that the higher FSS for adjusted radar data appears especially at the events from 2005. The same is valid for corrected data but the difference is significantly smaller.

The results shown in Figs. 1, 2, and 3 refer to $3 \mathrm{~h}$ rainfalls. Similar conclusions can be drawn for the other accumulation periods. Increasing accumulation gives larger standard deviation of difference between data types. As the rainfalls increase with accumulation, selection of thresholds on the quantile basis will be suitable.
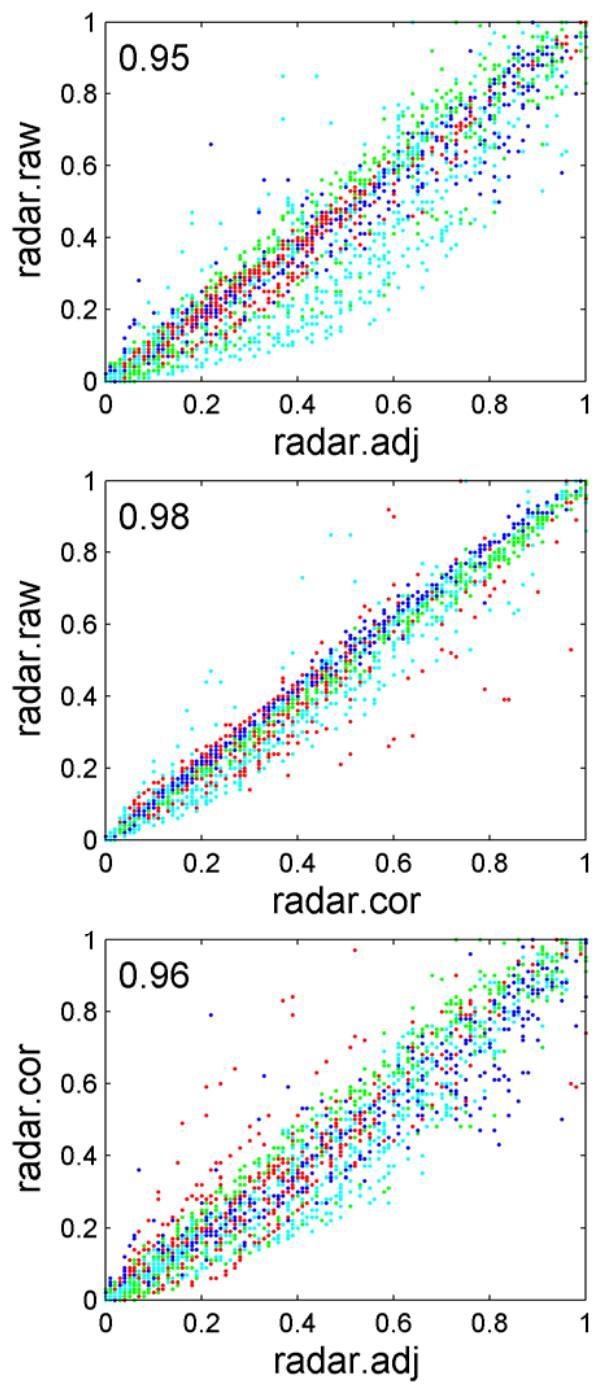

Fig. 3. Scatterplots of the FSS values for $3 \mathrm{~h}$ rainfalls and various radar-based data types. Each graph includes FSS values from all events, integration times, scales, and threshold values (1, 2, 5, $10 \mathrm{~mm}$ ). The set of FSS values correspond to the mean FSS from Fig. 2 (black curves). Single color refer to the events as follow: red - 13 July 2002, green - 15 July 2002, blue - 10 June 2004, and cyan - 23 May 2005. Correlations are given in each panel.

\section{Conclusions}

Three different radar-based QPE data sets were used for the QPF verification and the verification results were compared. The QPE data sets comprised of: (a) raw radar-based rainfall values, (b) gauge corrected radar-based rainfalls with a simple domain-wide correction, and (c) radar-based rainfalls with a pixel related gauge adjustment. As expected, the FSS values increased with decreasing rainfall thresholds and with increasing size of elementary area. This was valid for all verification data sets. 
The FSS resulting from area corrected radar and adjusted radar are larger than the FSS following from raw data set, in general. They confirm the well-known need to correct the raw rainfall values. There is only a small difference between the FSS values obtained by using radar-corrected and radaradjusted verification data. The radar adjustment gives mostly the largest FSS values and the difference between raw radar and corrected radar is smaller than the difference between raw radar and adjusted radar. However, the differences are only of the order of 0.01 and the FSS values based on various verification data are strongly correlated. We can conclude that the simple radar data correction can be applied at the QPF verification without pronounced deviation of FSS values from the detailed adjustment.

Nevertheless, the differences between FSS values are event dependent and increasing number of events with wide spectrum of FSS values would be useful for the generalization of the results. More extensive data set will be used in the next work.

Acknowledgements. The work was supported by the Ministry of Education Sports and Youths CR in the project OC112 (COST 731) and by the grants GAAV IAA300420804 and GACR 205/07/0905. We acknowledge the CHMI for the meteorological data support and the German Weather Service for the provision of COSMO code for research. The authors thank to Zbynek Sokol for the provision of locally adjusted radar data.

Edited by: S. C. Michaelides

Reviewed by: two anonymous referees

\section{References}

Casaioli, M., Mariani, S., Accadia, C., Gabella, M., Michaelides, S., Speranza, A., and Tartaglione, N.: Radar adjusted data versus modelled precipitation: a case study over Cyprus, Adv. Geosci., 7, 85-90, 2006, http://www.adv-geosci.net/7/85/2006/.

Ebert, E. E.: Fuzzy verification of high resolution gridded forecasts: a review and proposed framework, Meteorol. Appl., 15, 51-64, 2008.

Novak, P.: The Czech Hydrometeorological Institute's severe storm nowcasting system, Atmos. Res., 83, 450-457, 2007.

Rezacova, D., Sokol, Z., and Pesice, P.: A radar-based verification of precipitation forecast for local convective storms. Atmos. Res. 83, 211-224, 2007.

Rezacova, D., Zacharov, P., and Sokol, Z.: Uncertainty in the arearelated QPF for heavy convective precipitation, Atmos. Res., 93, 238-246, 2009.

Roberts N. M. and Lean H. W.: Scale-selective verification of rainfall accumulations from high-resolution forecasts of convective events, Mon. Weather. Rev., 136, 78-97, 2008.

Sokol, Z.: The use of radar and gauge measurements to estimate areal precipitation for several Czech river basins, Stud. Geophys. Geod. 47, 587-604, 2003.

Tartaglione, N., Mariani, S., Accadia, C., Casaioli, M., Gabella, M., Michaelides, S. C., and Speranza, A.: Sensitivity of forecast rainfall verification to a radar adjustment technique, Meteorol. Z., 15, 537-543, 2006.

Zacharov, P., Šálek, M., and Novák, P.: Comparison of different methods for estimating precipitation based on radar and rain gauge measurements (in Czech with English summary), Meteorol. Zpravy., Meteorol. Bull., ISSN 0026-1173, 57, 157-167, 2004.

Zacharov, P. and Rezacova, D.: Using the fractions skill score to assess the relationship between an ensemble QPF spread and skill, Atmos. Res., 94, 684-693, 2009. 